\title{
Influence of the hypothalamic paraventricular nucleus (PVN) on heart rate variability (HRV) in rat hearts via electronic lesion $^{1}$
}

\author{
Xin Deng ${ }^{\mathrm{a}}$, Xuhui Feng ${ }^{\mathrm{b}}$, Sen $\mathrm{Li}^{\mathrm{c}}$, Ya Gao ${ }^{\mathrm{d}}$, Bingzhi $\mathrm{Yu}^{\mathrm{e}}$ and Gensong $\mathrm{Li}^{\mathrm{a}, \mathrm{b}, *}$ \\ ${ }^{a}$ The Experimental Center of Functional Subjects, College of Basic Medical Scientific Research, China \\ Medical University, P.R. China \\ ${ }^{b}$ Department of Physiology, College of Basic Medical Scientific Research, China Medical University, \\ P.R. China \\ ${ }^{c}$ Department of Neurology, The First Affiliated Hospital, China Medical University, P.R. China \\ ${ }^{d} 96$ K 7-years, China Medical University, P.R. China \\ ${ }^{e}$ Department of Biochemistry and Molecular Biology, College of Basic Medical Scientific Research, \\ China Medical University, P.R. China
}

\begin{abstract}
Previous literatures have indicated that hypothalamic paraventricular nucleus (PVN) neurons are important for regulating the level of sympathetic and vagal nervous activity. Sympathovagal balance is closely related to heart rate variability (HRV). However, it still requires further elucidation regarding the effect of PVN on HRV by regulating sympathovagal balance. To detect the influence of the PVN on HRV, we evaluated the changes in time domain (including standard deviation of the R-R intervals (SDNN), and the root mean square of successive differences (RMSSD)) and frequency domain (including low frequency (LFnu), high frequency (HFnu) and the ratio of LF/HF) in HRV upon appropriate electronic stimulation, and lesions on the PVN of the rat in vivo. Cardiac vagal modulation was evaluated by HFnu; sympathetic modulation was evaluated by LFnu. Sympathovagal balance was evaluated by LF/HF and SDNN. Upon electronic stimulating (less than $0.6 \mathrm{~mA}$ ) to the PVN of rats, we found that LFnu and HFnu changed correspondingly but recovered after the stimulation. When the PVN of the rats was injured, the RR intervals were enhanced with the rats' unilaterally or bilaterally injured PVN, especially the bilateral lesion. Meanwhile, LFnu, LF/HF and SDNN decreased gradually, accompanied with an increase of HFnu levels. So these PVN changes may indicate alterations of the sympathovagal balance.
\end{abstract}

Keywords: Heart rate variability (HRV), paraventricular nucleus (PVN), Rat, electrical lesion

\footnotetext{
${ }^{1}$ Xin Deng and Xuhui Feng equally contributed to this article.

This work was supported by the National Nature Science Foundation of China Grants (No. 81370712 and 81270654) and no other potential conflict of interest relevant to this article was reported.

* Address for correspondence: Gensong Li, The Experimental Center of Functional Subjects, College of Basic Medical Scientific Research, China Medical University, P.R. China. Tel.: 0086(0)13386885803; E-mail: gsli@mail.cmu.edu.cn.
} 


\section{Introduction}

Automaticity is an intrinsic character of myocardiac cells. Their electrophysiological activity is largely modulated by the autonomic nervous system (ANS) $[1,2]$. The sympathetic nervous system (SNS) and parasympathetic (also called vagal in cardiovascular) nervous system (PNS) are the two branches of the ANS. Both branches may influence each other in response to the function of the central nervous system (CNS) [3]. Therefore, the ANS is critical to the physiological and pathological status of the myocardiac cells [4].

The paraventricular nucleus (PVN) of the hypothalamus is inter-connected with the other parts of the brain involved in cardiovascular function. As the central regulator of the cardiovascular system, the PVN is an important site that integrates the automaticity and response of the cardiovascular system [5-7]. Thus, the activities of the PVN and ANS may be tightly connected.

Recent studies demonstrated an effective computational method of heart rate variability (HRV) to reveal both cardiac sympatho-vagal modulation [3, 8-10]. HRV is a noninvasive measurement that assesses the autonomic modulation of cardiac activity. It may analyze beat-to-beat variability in normal R-R (NN) intervals in an ECG. In a normal heart, there are continuous physiological variations of the sinus cycles reflecting a balanced sympatho-vagal state by HRV.

The standard clinically used parameters of HRV consist of the time domain indices, geometric measures and frequency domain indices [11]. The time domain index includes the standard deviation of all NN intervals (SDNN), and the root mean square of successive differences (RMSSD) represents the changes of heart rate over time or over the RR intervals, which is the time between two successive $\mathrm{R}$ waves in QRS complexes. Geometric methods are from the conversion of sequences of normalized $\mathrm{RR}(\mathrm{NN})$ intervals. The power spectrum of the frequency domain included mainly a very low frequency (VLF), a low frequency (LF) and a high frequency (HF). LF is modulated by sympatho-vagal nervous systems. When the LF is expressed in normalized units (nu), the increase of the LFnu component (including tilt, mental and/or physical stress, and sympathetic pharmacological agents) is generally considered a consequence of sympathetic activity. The HFnu and RMSSD are generally defined as markers of vagal modulation, which is controlled by breathing frequency. The LF/HF ratio and SDNN reflect the global sympatho-vagal balance, so they may be used as a measure for the balance $[4,12]$. Although the parameters of HRV may reflect distinctly the influence of sympathetic or vagus nerves on the heart, the relationship between HRV and the ANS mediated by the PVN has not yet been fully elucidated.

To identify the effect of the PVN on HRV, we examined the changes of SDNN, LFnu, HFnu and LF/HF under the conditions of unilateral or bilateral PVN lesions over the course of time. We speculated that the PVN may play an important role on the balance of $\mathrm{LF} / \mathrm{HF}$, and it may regulate HRV through the ANS.

\section{Material and methods}

\subsection{Animals}

All experiments were performed at China Medical University (CMU) in accordance with the NIH Guidelines of the USA for Care and Use of Laboratory Animals. Protocols for animal handling and treatment procedures were reviewed and approved by the CMU Animal Care and Use Committee. Fifty male Wister rats (200-250 g) were obtained from the Department of Laboratory Animals, CMU. 
Two groups of rats were caged under controlled temperature $\left(24 \pm 2^{\circ} \mathrm{C}\right)$ with a dark/light cycle of $12 \mathrm{~h}$. All efforts were made to minimize animal suffering as well as the number of animals used.

\subsection{Successive ECG of rat under awake state}

Two days before the operation, all rats in an awake state were detected for successive ECG. Rats were placed in experimental cages. The ECG lead II was recorded with surface electrodes (ADInstruments, Australia). Measurements were collected at baseline, prior to electronic lesion and after the $4^{\text {th }}$ day, $7^{\text {th }}$ day and $14^{\text {th }}$ day of lesions. The conditions for the experiment are as following: sample rate, $1 \mathrm{k} / \mathrm{s}$; range, $10 \mathrm{mv}$; low pass, $200 \mathrm{~Hz}$; high pass, $10 \mathrm{~Hz}$; and signaling requirement, successively intact ECG of 5 mins for each data.

\subsection{Surgical procedure}

Rats were individually caged without restraints for $1-2 \mathrm{~h}$ to adapt to the experimental conditions. Rats were then anesthetized with sodium pentobarbital (30-35 mg kg${ }^{-1}$, i.p.) and additional doses were given as needed. Then they were placed prone in a stereotaxic apparatus (Japan) with the incisor bar 5 $\mathrm{mm}$ below the interauaral line. The rats were implanted with two guide cannulas directed to the PVN (1.8 $\mathrm{mm}$ posterior to bregma, $0.5 \mathrm{~mm}$ lateral to midline and $7.6 \mathrm{~mm}$ below the surface). The guide implantation technique was adapted based on a previous work $[5,13]$.

An electronic stimulated needle was inserted into one of the paraventricular nuclei, as previously described $[13,14]$. The PVN was stimulated with $0.2,0.4$ and $0.6 \mathrm{~mA}$ for $60 \mathrm{~s}$ or was impaired by using DC $1 \mathrm{~mA}$ for $60 \mathrm{~s}$, either at the right only or the entire part, according to experimental procedures. A PVN without lesions was used as the control. In the groups of the Sham operation, rats were processed according to above operation, but without electronic stimulation. Rats were allowed recuperation for 3-5 days after the surgery. Successive ECGs for all animals according to standard II lead were collected for a minimum of $5 \mathrm{~min}$ in the morning of the $4^{\text {th }}$ day, $7^{\text {th }}$ day and $14^{\text {th }}$ day, from 8:00 to 12:00, when the animals were conscious.

\subsection{HRV measurements}

Heart rate variability (HRV) parameters were measured over $256 \mathrm{RR}$ intervals as previously described [15], with Powerlab ${ }^{\circledR}$ system (ADInstruments) and Chart 5.0 software over 256 R-R intervals. Any ectopic beats were automatically identified and discarded by the software before the analysis of the captured beats. Small-animal HRV software (nevrokard) was then used to identify HRV data. The parameters of HRV were all proposed by the Task Force of the European Society of Cardiology and the North American Society of Pacing and Electrophysiology [11].

The variances of frequency components were transformed to a normalized unit (nu), which is performed by the following formula (VLF is from 0.04 to $0.1 \mathrm{~Hz}$, LF is from 0.1 to $1 \mathrm{~Hz}$ and $\mathrm{HF}$ is from 1 to $3 \mathrm{~Hz}$ ).

$$
L F \text { or } H F \operatorname{norm}(n u)=\frac{\operatorname{LF} \text { or } H F\left(m s^{2}\right)}{\text { Total power }\left(m s^{2}\right)-\operatorname{VLF}\left(m s^{2}\right)} \times 100
$$

All samples' ECGs were collected and analyzed by PowerLab ${ }^{\circledR}$ with Chart ${ }^{\circledR}$ software Version 5.0 
to obtain the parameters of HRV. The normalized unit (nu) is useful when evaluating the effects of different interventions in the same subject (graded tilting) or when comparing subjects with major differences in total power.

\section{5. $H \&$ E staining of the coronal brain slice}

Rat brains were removed and post-fixed in $4 \%$ formalin for $24 \mathrm{~h}$ and then washed, dehydrated from alcohol to dimethybenzene, and paraffin-embeded. Coronal brain slices of the rats in unilateral lesion and/or bilateral lesion were made and stained by standard $H \& E$. Examination was performed by light microscope (TE2000-S Nikon, Japan) photograph.

\subsection{Statistics}

Data are analyzed statistically by one-way analysis of variance (ANOVA). The level of significance was set at $95 \%(P<0.05)$. The software used for analysis of the data was the Statistical Package for the Social Sciences (SPSS, Chicago, IL, USA), version 13.0 for Windows. The data shown are mean \pm SEM of at least ten independent experiments $(n=10)$.

\section{Results}

\subsection{Effect of PVN on power spectral density upon stimulation by electronic currents}

Rats' PVN were stimulated with currents of $0.2,0.4$ or $0.6 \mathrm{~mA}$. The normalized auto-power spectral density from right to left showed HF, LF and VLF, respectively, in Figure 1. When the PVN was stimulated with $0.2,0.4$ and $0.6 \mathrm{~mA}$ currents, the components of HF, LF and VLF changed correspondingly, and these changes backed down gradually after the stimulation. These results suggest that when the current stimulation was less than $0.6 \mathrm{~mA}$, the changes were reversible.

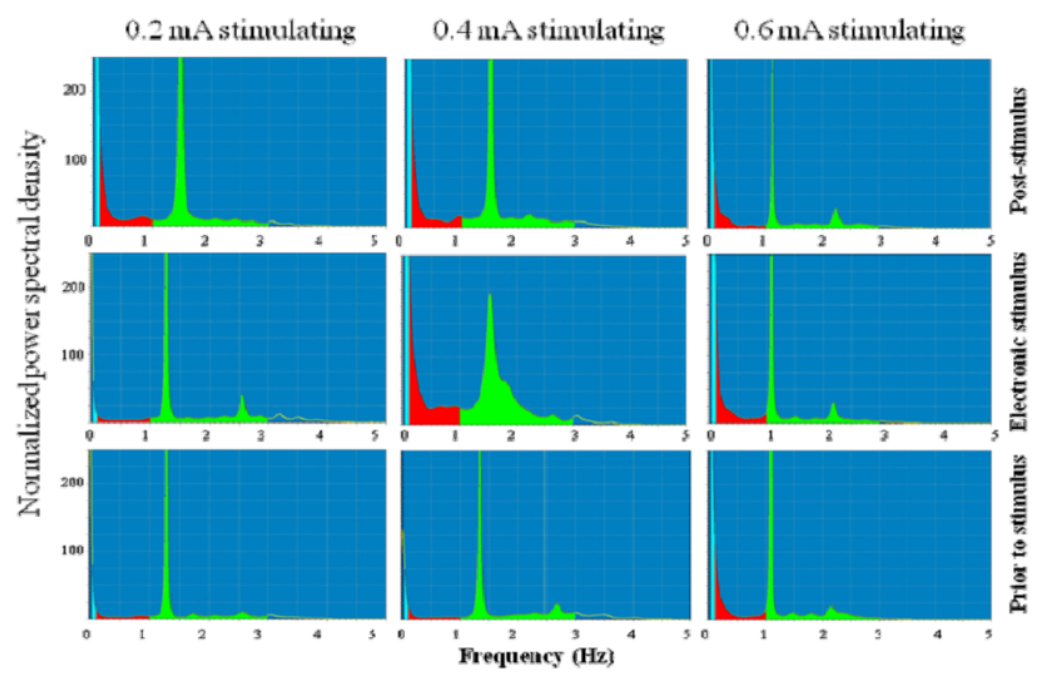

Fig. 1. Autoregressive spectrum of the PVN of the rats stimulated with different electronic currents. The right part is HF as indicated in green. The Middle is LF in red. The left part is VLF in ligh blue. When rat PVN was stimulated with $0.2 \mathrm{~mA}, 0.4$ $\mathrm{mA}$ or $0.6 \mathrm{~mA}$, the densities of VLF, LF and HF were shown in the frequence spectrogram, respectively. 


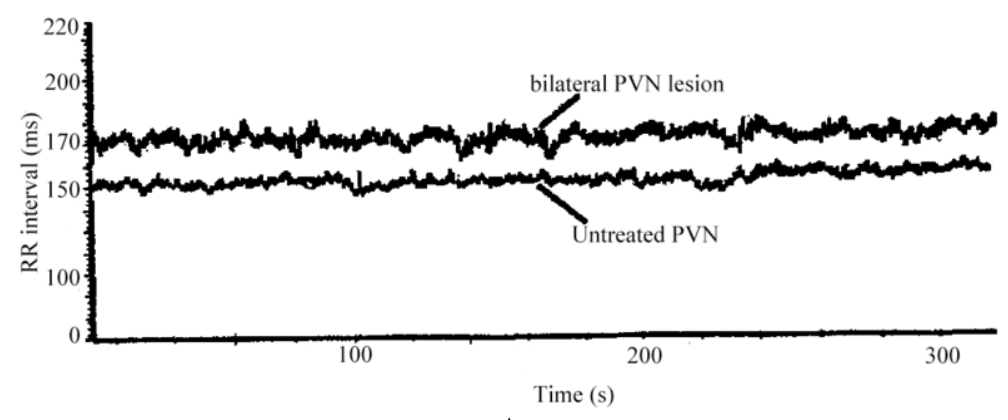

A

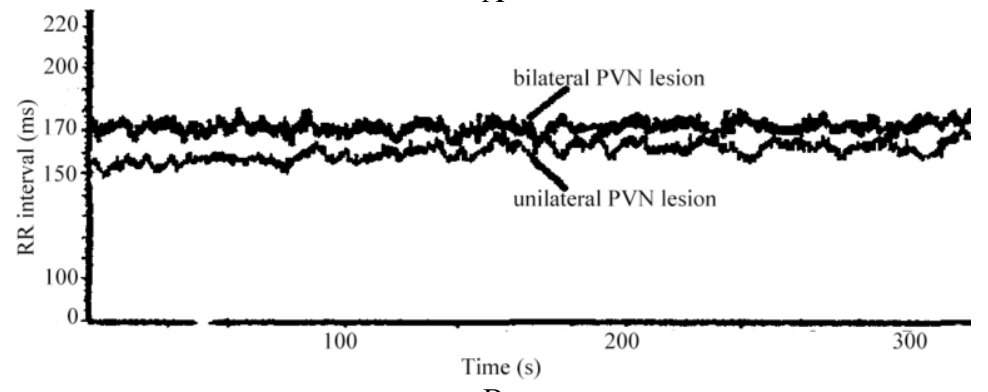

$\mathrm{B}$
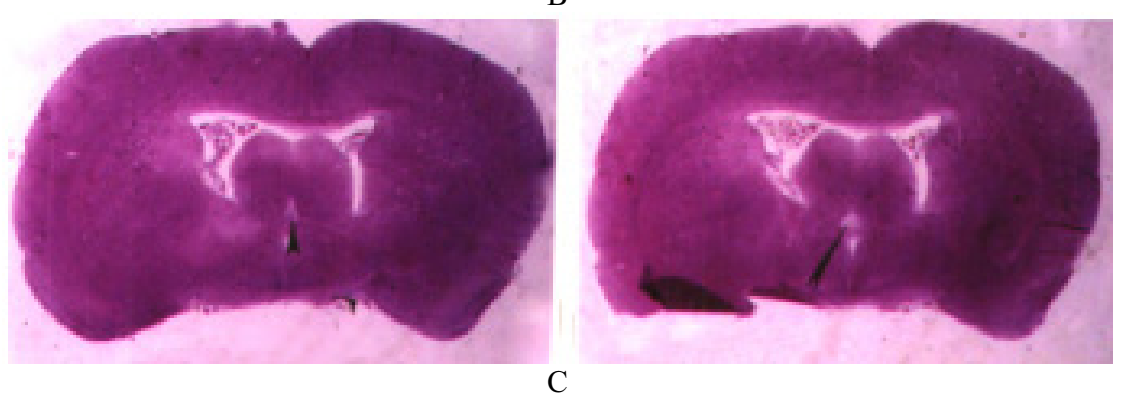

Fig. 2. Influence of PVN damage on RR intervals. A: RR interval in the PVN with bilateral lesion compared with that in the untreated PVN; B: RR interval in the PVN with bilateral lesion compared with that in the PVN with unilateral lesion; C: The localization of PVN lesions. Left: coronalis slice of rat brain at PVN with lesion at right; Right: coronalis slice of rat brain of PVN with bilateral lesion, indicated with the black arrowhead (1× magnification).

\subsection{Effect of Lesion of PVN on R-R chart}

Under the waking state, the heart rate (HR) of the rat over 5 minutes is about $460 \pm 45 \mathrm{~min}^{-1}$. Under a narcotic state, the HR is about $410 \pm 53 \mathrm{~min}^{-1}$ (data not shown). The data showed that no significant change in the $\mathrm{R}$ wave in both the waking state and the narcotic state. However, after the PVN was damaged by electric stimulation, the RR interval changed distinctly. The RR intervals of the normal group were shorter than that of the lesion group, especially that of the bilateral lesion group. The RR interval values of the normal, unilateral lesion, and bilateral lesion groups are $150 \pm 10 \mathrm{~ms}, 155 \pm 22 \mathrm{~ms}$ and $170 \pm 15 \mathrm{~ms}$, respectively (Figures $2 \mathrm{~A}$ and $2 \mathrm{~B}$ ). The heart rates also decreased in lesion group accordingly.

In the histology of the brain section, we observed that there was a non-staining area in the right of the PVN with a unilateral lesion PVN. In the PVN with a bilateral lesion, there was a triangle non-staining in the left and right areas of the PVN (Figure 2C, indicated by arrow). 


\subsection{Influence of PVN lesion on $H R V$}

Martins-Pinge et al. reported that the neurons within the PVN may contribute to cardiac autonomic modulation [5]. Our results showed that in the PVN with unilateral and/or bilateral lesions, the LFnu were much lower in postoperative day 4 , day 7 and day 14 than in the preoperative day $0(P<0.05)$, especially in day 14 for the PVN with a bilateral lesion $(P<0.05)$. During preoperative day 0 to postoperative day 4, the LFnu and SDNN in the PVN with unilateral and/or bilateral lesions had already gradually decreased with a significant difference $(P<0.05)$. After the postoperative day 7 , the decrease of LF gradually leveled off. As a result, there was no significant difference in LFun between postoperative day 7 and day $14(P>0.05)$. Furthermore, an opposite change in HFnu in contrast to LFnu and SDNN in the PVN with unilateral and/or bilateral lesion was observed (Figure 3). However, LF/HF and SDNN were similar to those of the LFun (Figure 3). Figure 4 is an illustration of the interaction of HRV, PVN and ANS.

\section{Discussion and Conclusion}

The areas of the central nervous system involved in cardiovascular control include the rostral

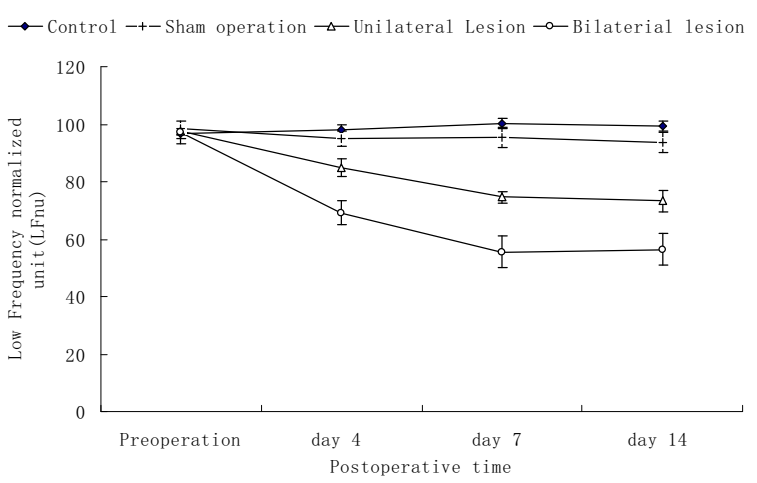

A

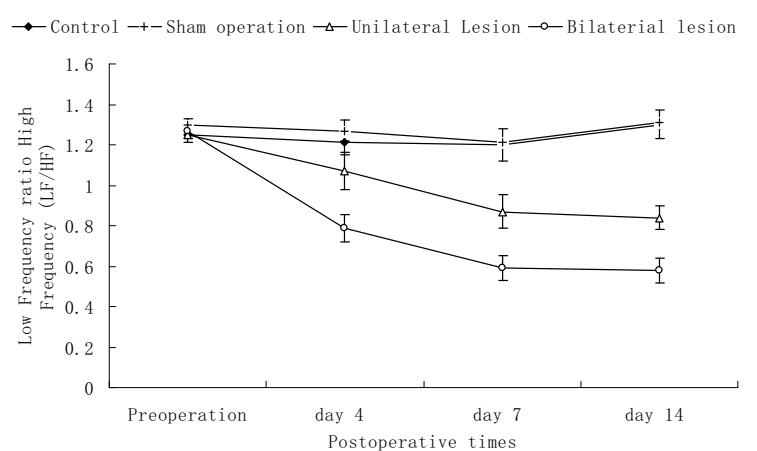

$\mathrm{C}$

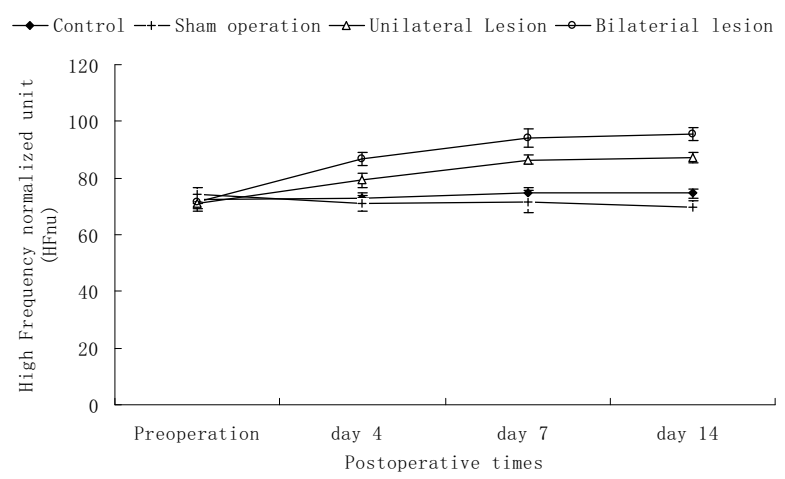

$\mathrm{B}$

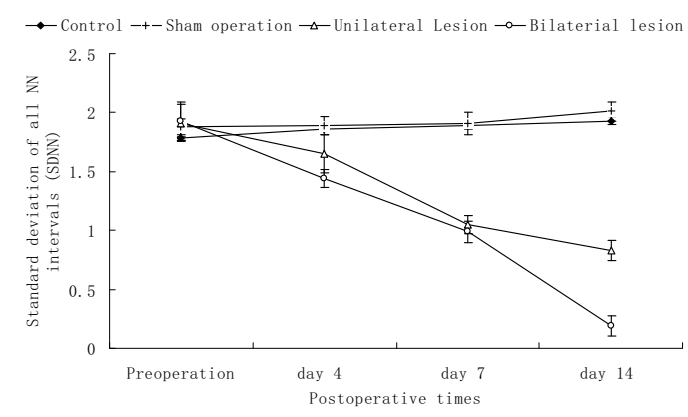

D

Fig. 3. Influence of PVN damage on HRV parameters in rat hearts. The standard deviation of the normalized RR intervals (SDNN), Low frequency normalized unit (LFnu), High frequency normalized unit (HFnu) and LF/HF were recorded in preoperation, postoperative 4, 7 and 14 days when the PVN was treated with electronic leision, respectively. Preoperation and sham operation groups were used as control. 


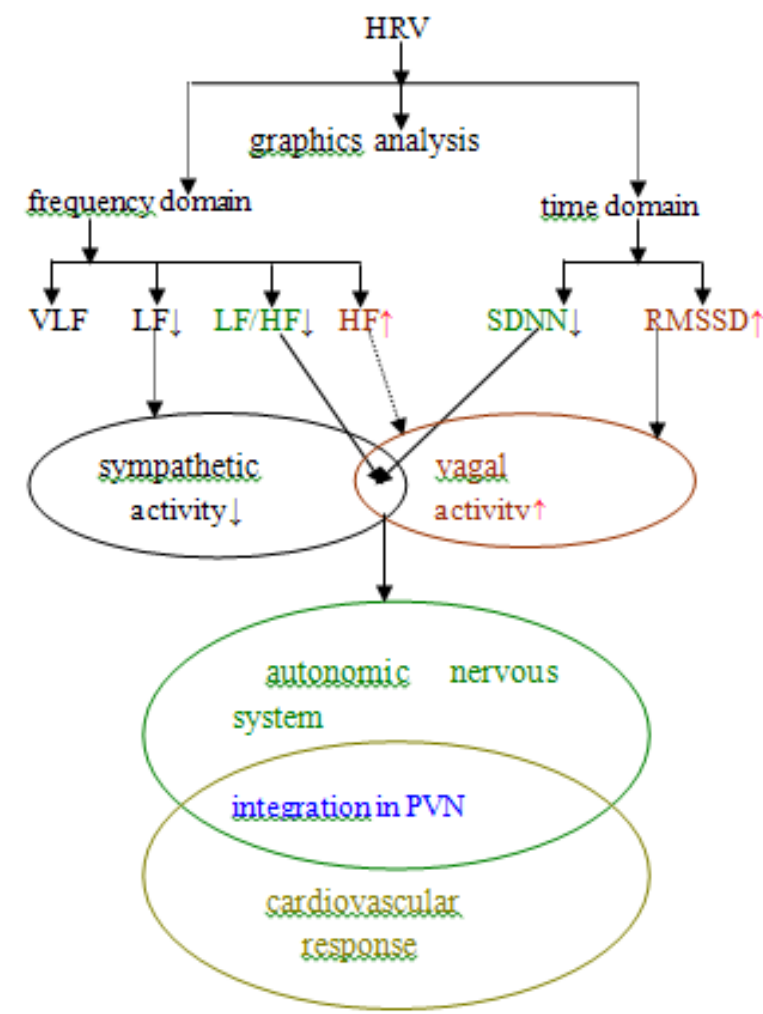

Fig. 4. Proposed diagram of the interactions among HRV, ANS and PVN. The HRV parameters of the heart consist of time domain (mainly including SDNN and RMSSD), geometric and frequency domain (mainly including VLF, LF, HF and $\mathrm{LF} / \mathrm{HF}$ ). LF is related to sympathetic activity. HF and RMSSD are related to vagal activity. LF/HF and SDNN are related to the sympathetic and vagal balance. ANS includes sympathetic and vagal nerves. Its function is integrated in PVN together with cardiovascular response.

ventrolateral medulla (RCLM) [16-24], the nucleus of solitary tract (NTS) [7, 25] and paraventricular nucleus (PVN) [26]. The PVN connects these areas in the brain involved in cardiovascular function, forming important neuroendocrine and autonomic modulation, which further modulates the homeostasis of the body through the brain stem, spinal cord, median eminence and posterior pituitary. A recent report by de Abreu indicated that neurons in the PVN could contribute to cardiac autonomic modulation by changing parasympathetic or sympathetic activity [5, 22, 23, 27]. Also, the interplay between the sympathetic and vagal outflows may be assessed by cardiovascular rhythmicity with appropriate spectral methodologies [28, 29].

The rat is the most simple and natural HRV model among vertebrates that are similar to human. There are few reports about the mechanism of the regulation of the PVN of rats, especially for events related to HRV. Here, we applied electronic stimulation and caused lesions on rats' PVN, and then investigated the changes in the spectral components by special HRV analysis.

We showed that electric-induced lesions in the PVN of rats results in decreases of LFnu, LF/HF and SDNN, but an increase of HF power and RMSSD of HRV, suggesting that these spectral components may be modified by PVN damage. In rats with a PVN lesion, the decrease of LFnu reflected the decline of sympathetic activity, which explains why the HRs slowed down gradually. Our data support the notion that the potential neurons and transmitters in the PVN may be closely related to HRV. A concrete procedure for this assumption should be further studied in this field. 


\section{Acknowledgment}

We are grateful to Associate Professor Frank Yang (Indiana University) for his help in English writing.

\section{References}

[1] N.J. Bramich, J.A. Brock and G.D.S Hirst, Potentiation by neostigmine of responses to vagal nerve stimulation in the sinus venosus of the toad, Autonomic Neuroscience 82 (2000), 109-114.

[2] Q.H. Chen and G.H. Toney, Excitability of paraventricular nucleus neurons that project to the rostral ventrolateral medulla is regulated by small-conductance $\mathrm{Ca}^{2+}$-activated $\mathrm{K}^{+}$channels, The Journal of Physiology 587 (2009), 4235-4247.

[3] J.G. Angela, S. Andrea, M. Francesca, M. Neal and M.T. Diane, Cardiac dysfunction and hypothalamic activation during a social crowding stressor in prairie voles, Autonomic Neuroscience: Basic and Clinic 156 (2007), 44-50.

[4] J. Sztajzel, Heart rate variability: A noninvasive electrocardiographic method to measure the autonomic nervous system, Swiss Medical Weekly 134 (2004), 514-522.

[5] R.B. Mastelari, H.C. de Souza, A. Lenhard, F.M. de Aguiar Corrêa and M.C. Martins-Pinge, Nitric oxide inhibition in paraventricular nucleus on cardiovascular and autonomic modulation after exercise training in unanesthetized rats, Brain Research 1375 (2011), 68-76.

[6] J.P. Herman, O. Eyigor, D.R. Ziegler and L. Jennes, Expression of the ionotropic glutamate receptor subunit mRNAs in the hypothalamic paraventricular nucleus of the rats, The Journal of Comparative Neurology 422 (2000), 352-362.

[7] L.C. Michelini and J.E. Stern, Exercise-induced neuronal plasticity in central autonomic networks: Role in cardiovascular control, Experimental Physiology 94 (2009), 947-960.

[8] B. Gutin, C.A. Howe, M.H. Johnson and M.C. Humphiries, Heart rate variability in adolescents: Relations to physical activity, fitness, and adiposity, Medicine and Science in Sports and Exercise 37 (2005), 1856-1863.

[9] S. Suzuki, S. Yanagita, S. Amemiya, Y. Kato, N. Kubota, et al., Effects of negative air ions on activity of neural substrates involved in autonomic regulation in rats, International Journal of Biometeorology 52 (2008), 481-489.

[10] B.G. Wallin and N. Charkoudian, Sympathetic neural control of integrated cardiovascular function: Insights from measurement of human sympathetic nerve activity, Muscle Nerve 36 (2007), 595-614.

[11] Task Force of the European Society of Cardiology and the North American Society of Pacing and Electrophysiology, Heart rate variability: standards of measurement, physiological interpretation and clinical use, Circulation 93 (1996), 1043-1065.

[12] J.O. Schwab, G. Eichner, H. Schmitt, S. Weber, M. Coch, et al., The relative contribution of the sinus and AV node to heart rate variability, Heart 89 (2003), 337-338.

[13] A.R. Zahran, P. Vachon, F. Courtois and S. Carrier, Increases in intracavernous penile pressure following injections of excitatory amino acid receptor agonists in the hypothalamic paraventricular nucleus of anesthetized rats, The Journal of Urology 164 (2000), 1793-1797.

[14] J. Xiao, J.B. Levitt and R. Buffenstein, A stereotaxic atals of the brain of the naked mole-rat (Hererocephalus galber), Neuroscience 141 (2006), 1415-1435.

[15] X. Wang, J.F. Thayer, F. Treiber and H. Snieder, Ethnic differences and heritability of heart rate variability in Africanand European American youth, The American Journal of Cardiology 96 (2005), 1166-1172.

[16] M.C. Martins-Pinge, L.K. Becker, M.R.L. Garcia, D.B. Zoccal, R.V. Neto, et al., Attenuated pressor responses to amino acids in the rostral ventrolateral medulla after swimming training in conscious rats, Autonomic Neuroscience: Basic \& Clinical 122 (2005), 21-28.

[17] L.K. Becker, R.A. Santos and M.J. Campagnole-Santos, Cardiovascular effects of angiotensin II and angiotensin-(1-7) at the RVLM of trained normotensive rats, Brain Research 1040 (2005), 121-128.

[18] P.J. Mueller, Exercise training attenuates increases in lumbar sympathetic nerve activity produced by stimulation of the rostral ventrolateral medulla, Journal of Applied Physiology 102 (2007), 803-813.

[19] S.B. DeAbreu, A. Lenhard, A. Mehanna, H.C. de Souza, F.M. Correa, et al., Role of paraventricular nucleus in exercise training-induced autonomic modulation in conscious rats, Autonomic Neuroscience: Basic \& Clinical 148 (2009), 28-35.

[20] A. Akine, M. Montanaro and A.M. Allen, Hypothalamic paraventricular nucleus inhibition decreases renal sympathetic nerve activity in hypertensive and normotensive rats, Autonomic Neuroscience: Basic \& Clinical 108 (2003), 17-21.

[21] M.R. Zahner and H.L. Pan, Role of paraventricular nucleus in the cardiogenic sympathetic reflex in rats, American Journal of Physiology, Regulatory, Integrative and Comparative Physiology 288 (2005), R420-R426. 
[22] J.L. Segar, D.L. Ellsbury and O.M. Smith, Inhibition of sympathetic responses at birth in sheep by lesion of the paraventricular nucleus, American Journal of Physiology, Regulatory, Integrative and Comparative Physiology 283 (2002), R1395-1403.

[23] N. Montano, A. Porta, C. Cogliati, G. Costantino, E. Tobaldini, et al., Heart rate variability explored in the frequency domain: A tool to investigate the link between heart and behavior, Neuroscience and Biobehavioral Reviews 33 (2009), 71-80.

[24] L. Cipryan and M. Litschmannova. Intra-day and inter-day reliability of heart rate variability measurement, Journal of Sports Sciences 31 (2013), 150-158.

[25] P.J. Mueller and E.M. Hasser. Putative role of the NTS in alterations in neural control of the circulation exercise training in rats, American Journal of Physiology, Regulatory, Integrative and Comparative Physiology 290 (2006), R383-R392.

[26] S. Gurtu, K.K. Pant, J.N. Sinha and K.P. Bhargava, An investigation into the mechanism of cardiovascular responses elicited by electrical stimulation of locus coeruleus and subcoeruleus in the cat, Brain Research 301 (1984), 59-64.

[27] R.B. Mastelaria, Bagolan de Abreu, F. Morgan de Aguiar Corrêa, H.C. Dutra de Souza and M.C. Martins-Pinge, Glutamatergic neurotransmission in the hypothalamus PVN on heart rate variability in exercise trained rats, Autonomic Neuroscience 170 (2012), 42-47.

[28] L.H. Chang, T.C. Ma, S.L. Tsay and G.P. Jong, Relationships between pain intensity and heart rate variability in patients after abdominal surgery: A pilot study, Chinese Medical Journal 125 (2012), 1964-1969.

[29] E.V. Kurjanova, D.L. Teplyj and N.V. Zereninova, Development of regulation of the cardiac chronotropic function in albino rats during the early postnatal ontogeny according to the results of spectral analysis of heart rhythm variability, Bulletin of Experimental Biology and Medicine 152 (2012), 675-678. 